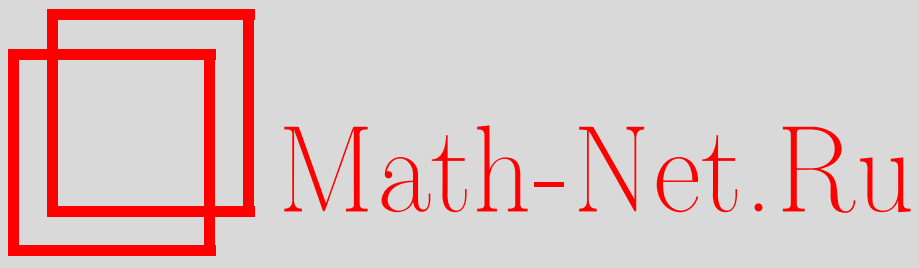

В. Е. Зотеев, Построение разностных уравнений для повышения точности параметрической идентификации колебательных систем со слабой нелинейностью общего вида, Вестн. Сам. гос. техн. ун-та. Сер. Физ.-мат. науки, 2000, выпуск 9, 169173

DOI: https://doi.org/10.14498/vsgtu44

Использование Общероссийского математического портала Math-Net.Ru подразумевает, что вы прочитали и согласны с пользовательским соглашением

http://www . mathnet.ru/rus/agreement

Параметры загрузки:

IP : 3.89.185.249

26 апреля 2023 г., 15:20:58 


\section{ПОСТРОЕНИЕ РАЗНОСТНЫХ УРАВНЕНИЙ ДЛЯ ПОВЫШЕНИЯ ТОЧНОСТИ ПАРАМЕТРИЧЕСКОЙ ИДЕНТИФИКАЦИИ КОЛЕБАТЕЛЬНЫХ СИСТЕМ СО СЛАБОЙ НЕЛИНЕЙНОСТЬЮ ОБЩЕГО ВИДА}

Рассматриваются принципы построения разностных уравнений колебаний систем со слабой нелинейностью общего вида, обеспечивающих существенное повышение точности оценок динамических характеристик механических систем за счет устранения погрешности аппроксимации исходного нелинейного дифференциального уравнения конечно-разностным. Приводятся результаты численного эксперимента, подтверждающего эффективность предлагаемого подхода к построению линейных дискретных моделей колебаний нелинейных диссипативных систем.

Известно, что долговечность и надежность различных механических систем (МC) непосредственно связана с их динамическими характеристиками (ДХ) [1]. О техническом состоянии $\mathrm{MC}$ можно судить по изменению ее диссипативных или жесткостных характеристик в процессе эксплуатации, прочностных или других видов испытаний. Повышение точности и достоверности оценок ДХ требует непрерывного усовершенствования математических моделей и разработки на их основе новых алгоритмов идентификации МС [2] Одной из основных проблем при этом является построение математических моделей, с одной стороны, адекватно аппроксимирующих наблюдаемый динамический процесс в системе, а с другой - сводящих процедуру вычисления ДХ к решению корректно поставленной обратной задачи математической физики [3]. Без учета этих аспектов ни один алгоритм оценивания не может считаться работоспособным.

Большой класс МС образуют системы с малой диссипацией (рассеянием) энергии колебаний. К таким системам можно отнести детали и узлы отдельных и взаимодействующих механизмов, а также конструкционные материалы, применяемые в машиностроении. Уравнение, описывающее свободные колебания таких систем, имеет вид

$$
m y^{\prime \prime}(t)+c y(t)=-b f\left[y(t), y^{\prime}(t)\right],
$$

где $m$ и $c$ - масса и жесткость системы; $-b f\left[y(t), y^{\prime}(t)\right]$ - произвольная нелинейная сила, обуславливающая, в общем случае, как диссипацию энергии колебаний, так и нелинейный характер упругого деформирования звеньев механизма, в частности, гистерезисные явления в реальных механических системах; $b$ - малый параметр, удовлетворяющий условию $b<<2 \sqrt{\mathrm{cm}}$.

Динамический процесс в этих системах описывается функцией $y(t)$, которая является решением уравнения (1). При заданных коэффициентах в дифференциальном уравнении и начальных условиях его решение может быть получено с заданной точностью одним из численных методов, например, с помощью многошаговых методов Адамса-Бэшфорта или АдамсаМултона [4]. Точность полученного на дискретном множестве приближенного решения $\tilde{y}(\tau k)$, $k=0,1,2, \ldots$, зависит от шага интегрирования $\tau$ и порядка используемого метода [4]. При уменьшении $\tau$ погрешность аппроксимации уменьшается.

Динамические характеристики системы определяются через коэффициенты ДУ следующим образом: $\omega_{0}=\sqrt{\frac{c}{m}}-$ собственная частота системы; $\quad \delta(a)=\frac{b}{m \omega_{0} a^{2}} \int_{0}^{T} f\left[y(t), y^{\prime}(t)\right] d t \quad-$ декремент колебаний, соответствующий амплитуде колебаний $a$. При вычислении коэффициентов ДУ $\frac{c}{m}$ и $\frac{b}{m}$ фактически решается обратная задача: нахождение параметров уравнения по известным значениям $y_{k}$, вычисленным в моменты времени $t_{k}=\tau k, k=0,1,2, \ldots$. Для ее решения исходное ДУ заменяется конечно-разностным уравнением, причем погрешность аппроксимации зависит как от порядка точности применяемых формул численного дифференцирования, так и от выбранного шага дискретизации $\tau$. Например, при использовании известных формул численного дифференцирования второго порядка точности уравнение (1) аппроксимируется разностным уравнением вида 


$$
\alpha_{1} y_{k-1}+\alpha_{2} F\left(y_{k}, y_{k-2}\right)=y_{k}+y_{k-2}, \quad k=2,3, \ldots
$$

где $\alpha_{1}=2-\left(\omega_{0} \tau\right)^{2}, \alpha_{2}=-\frac{b \tau^{2}}{m} \quad$ - коэффициенты разностного уравнения, известным образом связанные с параметрами уравнения (1).

Вычисление коэффициентов $\alpha_{1}$ и $\alpha_{2}$ из (2) сводится к решению системы линейных алгебраических уравнений (СЛАУ), число которых определяется объемом выборки $\left\{y_{k}\right\}$, а устойчивость вычислений - обусловленностью матрицы нормальной системы. Очевидно, что одной из основных составляющих погрешности в оценках $\alpha_{1}$ и $\alpha_{2}$ является погрешность аппроксимации. Однако при уменьшении шага дискретизации резко ухудшается устойчивость вычислений при решении СЛАУ, т. е. нарушается корректность постановки математической задачи. В итоге результирующая ошибка не только не уменьшается, а напротив, начинает возрастать. Это проявляется тем сильнее, чем больше погрешность в результатах наблюдений $\left\{y_{k}\right\}$. Следовательно, применение разностных уравнений вида (2) при параметрической идентификации колебательных систем приводит к оценкам, точность которых заведомо ограничена и не может быть улучшена за счет изменения шага дискретизации $\tau$.

Для устранения этого существенного недостатка рассмотрим другой способ построения линейной дискретной модели для уравнения (1). Он основан на использовании приближенного, полученного с заданной точностью, инвариантного к $\tau$, аналитического решения ДУ (1). Малый параметр в уравнении позволяет на основе методов возмущений получать равномерно пригодные разложения решения ДУ с высокой степенью точности [5]. Так для уравнения (1) с учетом простого решения невозмущенной задачи равномерно пригодное разложение первого порядка имеет вид

$$
y(t)=a(t) \cos [\omega t+\psi(t)],
$$

где функции $a(t)$ и $\psi(t)$ могут быть найдены, например, методом многих масштабов или методом усреднения [5]. Переходя от аналитического решения (3) к дискретной функции, можно затем построить линейную дискретную модель (ЛДМ), линейно связывающую некоторые функции от трех последовательных отсчетов $y_{k}, y_{k-1}, y_{k-2}$. При этом эффективным инструментом часто оказывается применение $Z$ - преобразования [6]. В полученной таким образом ЛДМ

$$
\lambda_{1} F_{1}\left(y_{k}, y_{k-1}, y_{k-2}\right)+\lambda_{2} F_{2}\left(y_{k}, y_{k-1}, y_{k-2}\right)+\ldots+\lambda_{r} F_{r}\left(y_{k}, y_{k-1}, y_{k-2}\right)=F_{r=1}\left(y_{k}, y_{k-1}, y_{k-2}\right)
$$

коэффициенты $\lambda_{i}$ известным образом связаны с коэффициентами уравнения (1) и находятся из решения соответствующей СЛАУ. Достоинством такой модели является то, что вычисленные на ее основе оценки ДХ системы содержат не погрешность аппроксимации, а погрешность равномерно пригодного разложения, которая не зависит от $\tau$ и может быть сделана сколь угодно малой за счет увеличения числа членов разложения [5]. Поэтому при использовании такой модели нет необходимости стремиться к малым $\tau$ и тем самым провоцировать ухудшение устойчивости вычисления оценок ДХ. Это приводит к устранению одной из основных составляющих погрешности вычисления параметров МС и, следовательно, к существенному повышению точности.

Рассмотрим предлагаемый подход к решению задачи параметрической идентификации колебательных систем со слабой нелинейностью на примере построения и исследования эффективности линейной дискретной модели свободных колебаний систем с турбулентным (гидродинамическим) трением [7,8]. Дифференциальное уравнение, описывающее свободные колебания систем с турбулентным трением, имеет вид

$$
m y^{\prime \prime}(t)+b y^{\prime}\left|y^{\prime}\right|+c y(t)=0 .
$$

Применение формул численного дифференцирования второго порядка точности позволяет аппроксимировать это уравнение разностным уравнением

$$
\alpha_{1} y_{k-1}+\alpha_{2}\left(y_{k}-y_{k-2}\right)\left|y_{k}-y_{k-2}\right|=y_{k}+y_{k-2},
$$

коэффициенты которого $\alpha_{1}$ и $\alpha_{2}$ связаны с параметрами ДУ (5) формулами $\alpha_{1}=2-\left(\omega_{0} \tau\right)^{2}, \alpha_{2}=-\frac{b}{4 m}, \omega_{0}=\sqrt{\frac{c}{m}}-$ собственная частота системы; $\tau-$ шаг дискретизации по времени. 
Очевидно, что разностное уравнение (6) линейно относительно переменных $\alpha_{1}$ и $\alpha_{2}$, и, следовательно, для их вычисления могут быть использованы методы прикладного линейного регрессионного анализа [9]. Однако результат вычислений будет всегда содержать ошибку, связанную с погрешностью аппроксимации уравнения (5) разностным уравнением (6). Проведены исследования зависимости погрешности вычисления коэффициентов ДУ $\frac{c}{m}$ и $\frac{b}{m}$ от шага дискретизации $\tau$ при различной точности в исходных данных. Моделировался динамический процесс в системе с турбулентным трением с параметрами $\frac{c}{m}=39,4784$ и $\frac{b}{m}=0,00375$, что соответствует частоте колебаний $\omega=2 \pi$ и начальному декременту колебаний $\delta_{0}=0,01$. Использовалась выборка отсчетов $y_{k}$ объемом $N=21$, точные значения которых округлялись до 4,3 и 2 десятичных знаков после запятой. Результаты исследований представлены на рис. $1,2$.

На рис. 1 представлены зависимости погрешности вычисления коэффициента $\frac{b}{m}$. Кривая 1 соответствует наибольшей точности в исходных данных. Видно, что с увеличением $\tau$ погрешность монотонно возрастает. При этом в результирующей погрешности доминирующей является погрешность аппроксимации, а неустойчивость вычислений при малых $\tau$ практически не сказывается. Кривая 2 соответствует округлению значений $y_{k}$ до 3 десятичных знаков. В этом случае при $\tau>0,05$ также определяющей является погрешность аппроксимации, однако при малых $\tau$ на точность вычислений уже существенно влияет неустойчивость

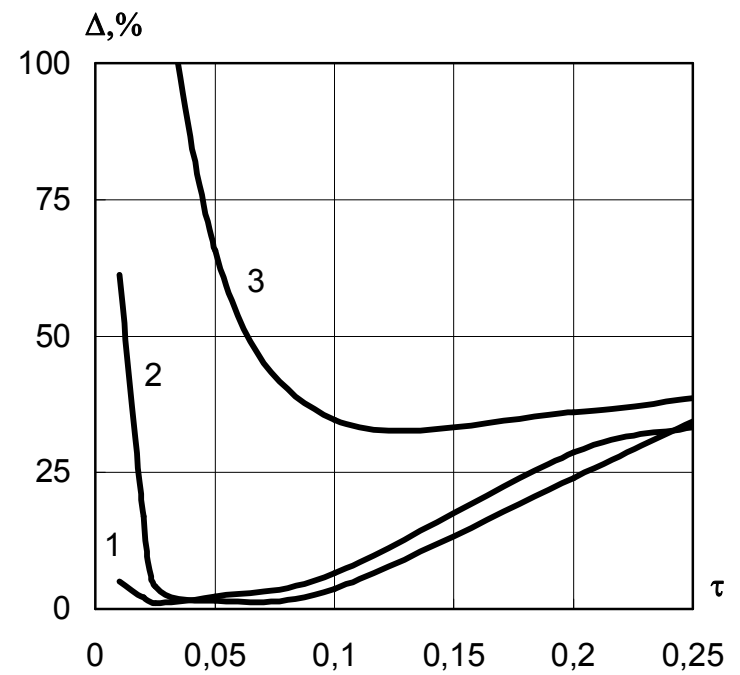

Р и с.1. Зависимости погрешности вычисления $\frac{b}{m}$ от шага дискретизации $\tau$ при аппроксимации дифференциального уравнения конечно-разностным уравнением второго порядка точности

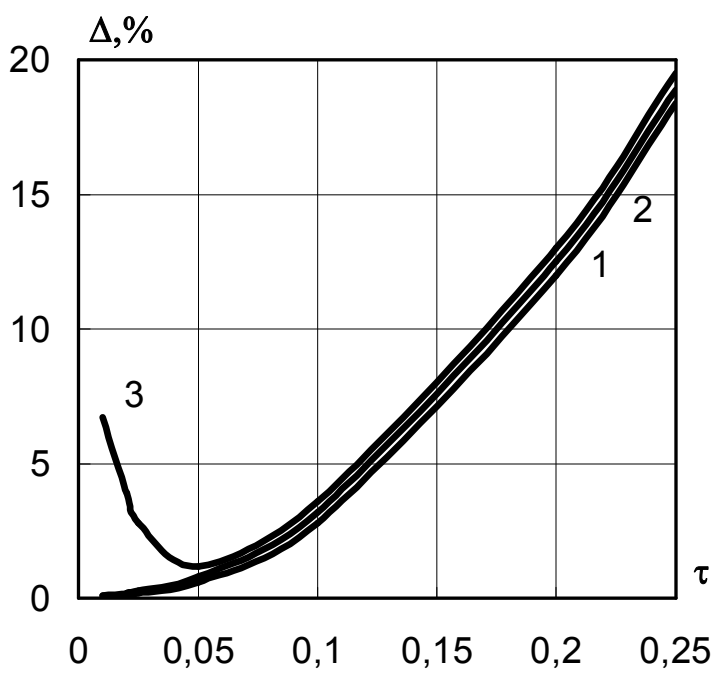

$\mathrm{P}$ и с.2. Зависимости погрешности вычисления $\frac{c}{m}$ от шага дискретизации $\tau$ при аппроксимации дифференциального уравнения конечно-разностным уравнением второго порядка точности

вычислений коэффициентов разностного уравнения (6). При погрешности в исходных данных, соответствующей реальной ситуации проведения научно-технического эксперимента (кривая 3 ) результирующая ошибка вычислений не менее $30-35 \%$ во всем диапазоне изменения $\tau$.

Аналогичные результаты получены и при исследовании зависимости погрешности вычисления коэффициента $\frac{c}{m}$ от шага дискретизации. Соответствующие кривые изображены на рис.2.

При малом параметре $b$ в уравнении (5) можно построить его приближенное решение в виде равномерно пригодного разложения по малому параметру [5]. Равномерно пригодное раз- 
ложение первого порядка ищется в виде (3), где функции $a(t)$ и $\psi(t)$ находятся из решения системы дифференциальных уравнений

$$
\begin{aligned}
& a^{\prime}(t)=-\frac{b}{m T} \int_{0}^{2 \pi} a^{2}(t) \sin ^{2} \varphi|\sin \varphi| d \varphi ; \\
& a(t) \psi^{\prime}(t)=-\frac{b}{m T} \int_{0}^{2 \pi} a^{2}(t) \sin \varphi \cos \varphi|\sin \varphi| d \varphi .
\end{aligned}
$$

При достаточно малом $b$ функции $a(t)$ и $\psi(t)$ оказываются медленно меняющимися функциями времени, на интервале интегрирования они изменяются незначительно и, следовательно, в первом приближении на этом интервале их можно считать постоянными. С учетом этого из (7) и (8) получаем

$$
a(t)=\frac{a_{0}}{1+\frac{4 b \omega a_{0}}{3 \pi m} t} ; \quad \psi(t)=\psi_{0} .
$$

Таким образом, приближенное решение уравнения (5) имеет вид

$$
y(t)=\frac{a_{0}}{1+\frac{\delta_{0}}{T} t} \cos \left(\omega t+\psi_{0}\right),
$$

где $\delta_{0}=\frac{8 b a_{0}}{3 m}-$ начальный декремент колебаний; $T=\frac{2 \pi}{\omega}-$ частота колебаний; $a_{0}$ и $\psi_{0}-$ начальные амплитуда и фаза колебаний. Проведенные расчеты показали, что погрешность приближенного решения (10) при начальном декременте колебаний $\delta_{0} \leq 0,01$ не превышает $0,1 \%$.

На основе приближенного решения (10) строится линейная дискретная модель свободных колебаний систем с турбулентным трением. Переходя в (10) к дискретной функции на множестве $t_{k}=\tau k$, где $\tau$ - шаг дискретизации, можно получить равенство

$$
\left(1+\lambda_{1} k\right) y_{k}=a_{0} \cos \left(\omega \tau k+\psi_{0}\right)
$$

где $\lambda_{1}=\frac{\delta_{0} \tau}{T}$. Применяя к этому равенству $Z$-преобразование, получаем

$$
Z\left\{\left(1+\lambda_{1} k\right) y_{k}\right\}=a_{0} \frac{\cos \psi_{0}-z^{-1} \cos \left(\omega \tau-\psi_{0}\right)}{1-\lambda_{0} z^{-1}+z^{-2}},
$$

где $\lambda_{0}=2 \cos \omega \tau$. Из последнего соотношения после простых алгебраических преобразований и обратного $Z$-преобразования получаем

$$
\left(1+\lambda_{1} k\right) y_{k}-\lambda_{0}\left[1+\lambda_{1}(k-1)\right] y_{k-1}+\left[1+\lambda_{1}(k-2)\right] y_{k-2}=a_{0} \cos \psi_{0} \delta_{k}-a_{0} \cos \left(\omega \tau-\psi_{0}\right) \delta_{k-1},
$$

где $\delta_{k}$ - символ Кронекера. При $k=0$ и 1 из (11) можно получить формулы для вычисления начальных амплитуды и фазы колебаний

$$
a_{0}=\sqrt{y_{0}^{2}+\frac{\left[2 y_{1}\left(1+\lambda_{1}\right)-y_{0} \lambda_{0}\right]^{2}}{4-\lambda_{0}^{2}}} ; \quad \psi_{0}=\arccos \frac{y_{0}}{a_{0}} .
$$

При $k \geq 2$ из (11) получаем линейную дискретную модель вида

$$
\lambda_{0} y_{k-1}+\lambda_{1}\left[k y_{k}+(k-2) y_{k-2}\right]+\lambda_{2}(k-1) y_{k-1}=y_{k}+y_{k-2},
$$

где $\lambda_{0}=2 \cos \omega \tau ; \quad \lambda_{1}=-\frac{\delta_{0} \tau}{T} ; \quad \lambda_{2}=-\lambda_{0} \lambda_{1}$.

Коэффициенты ЛДМ (13) $\lambda_{i}$ вычисляются из решения соответствующей системы линейных алгебраических уравнений одним из методов прикладного линейного регрессионного анализа [9]. Динамические характеристики системы с турбулентным трением находятся из формул

$$
\omega=\frac{1}{\tau} \arccos \frac{\lambda_{0}}{2} ; \quad \delta_{0}=-\frac{2 \pi \lambda_{1}}{\arccos \frac{\lambda_{0}}{2}},
$$




$$
\text { а коэффициенты ДУ (5) из выражений } \quad \frac{c}{m}=\omega^{2} ; \quad \frac{b}{m}=\frac{3 \delta_{0}}{8 a_{0}} \text {. }
$$

Проведенные исследования показали существенное преимущество модели (13), построенной по описанному выше алгоритму, по сравнению с конечно-разностным уравнением (6). На рис.3, 4 представлены зависимости погрешности вычисления коэффициентов $\frac{b}{m}$ и $\frac{c}{m}$ соответственно. Видно, что во всех трех случаях, соответствующих округлению исходных данных до 4,3 и 2 десятичных знаков после запятой (кривые 1,2 и 3), результирующая погрешность вычисления коэффициентов ДУ, начиная с $\tau>0,15$, не превышает $2 \%$ для первого коэффициен -

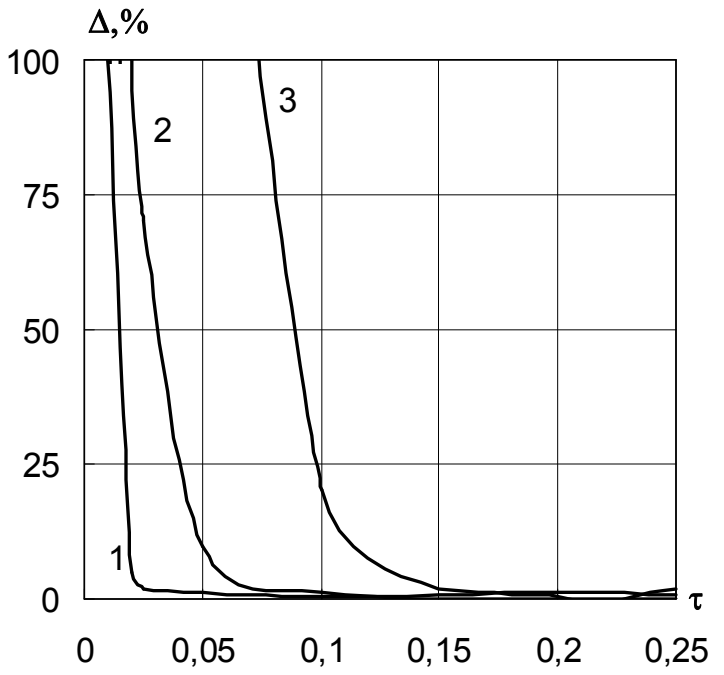

Р и с. 3. Зависимости погрешности вычисления $\frac{b}{m}$ от шага дискретизации $\tau$ при использовании линейной дискретной модели

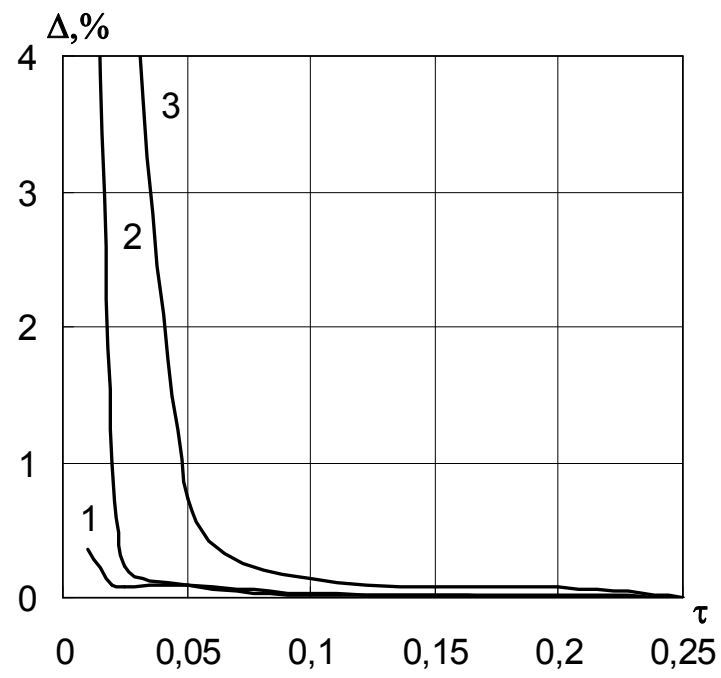

Р и с. 4. Зависимости погрешности вычисления $\frac{c}{m}$ от шага дискретизации $\tau$ при использовании линейной дискретной модели

та и $0,1 \%$ для второго. Это достигается тем, что в полученных оценках отсутствует погрешность аппроксимации, а погрешность приближенного решения (10) практически на результат не влияет. По сравнению с разностным уравнением (6) точность вычислений увеличивается на порядок.

Таким образом, рассмотренный подход к построению линейных дискретных моделей для задач параметрической идентификации колебательных систем со слабой нелинейностью общего вида позволяет существенно повысить точность оценок ДХ системы за счет устранения погрешности, связанной с аппроксимацией дифференциального уравнения конечно-разностным.

\section{БИБЛИОГРАФИЧЕСКИЙ СПИСОК}

1. Явленский К.Н., Явленский А.К. Вибродиагностика и прогнозирование качества механических систем. Л.: Машиностроение, 1983. $253 \mathrm{c}$.

2. Семенычев В.К., Зотеев В.Е. Определение параметров затухающих колебаний на основе разностных схем. // Проблемы прочности. 1988. № 12. С 101-105.

3. Марчук Г.И. Методы вычислительной математики. М.: Наука, 1977. 456 с.

4. Холл Д., Уатm Д. Современные численные методы решения обыкновенных дифференциальных уравнений. М.: Мир, 1979.

5. Найфэ А. Введение в методы возмущений: Пер. с англ. М.: Мир, 1984.

6. Деч Г. Руководство к практическому применению преобразования Лапласа и Z-преобразования. М.: Наука, 1971. $288 \mathrm{c}$.

7. Пановко А.Г. Основы прикладной теории колебаний и удара. Л. : Машиностроение, 1987. 224 с.

8. Зотеев B.E. Разработка и исследование линейных дискретных моделей колебаний диссипативных систем // Вестн. Самар. гос. техн. ун-та. Сер. Физ.- мат. науки. Вып.7. 1999. С.170-177.

9. Себер Дж. Линейный регрессионный анализ. М.: Мир, 1980. 456 с. 